\title{
The numerical method for computing the ground state of the two-component dipolar Bose-Einstein condensate
}

\author{
Si-Qi Li, Xiang-Gui Li* and Dong-Ying Hua
}

\section{"Correspondence:}

xianggui-li@vip.sina.com

School of Applied Science, Beijing

Information Science and

Technology University, Beijing,

100192, P.R. China

\section{Springer}

\begin{abstract}
A two-component Bose-Einstein condensate described by two coupled Gross-Pitaevskii (GP) equations in three dimensions is considered, where one equation has dipole-dipole interactions while the other one has only the usual s-wave contact interaction, in a harmonic trap. The singularity in the dipole-dipole interactions brings significant difficulties both in mathematical analysis and in numerical simulations. The backward Euler method in time and the sine spectral method in space are proposed to compute the ground states. Numerical results are given to show the efficiency of this method.
\end{abstract}

Keywords: Bose-Einstein condensate; Gross-Pitaevskii equation; dipolar interaction; ground state; spectral method

\section{Introduction}

Since 1995, the Bose-Einstein condensation (BEC) of ultra-cold atomic and molecular gases has attracted much attention both theoretically and experimentally. Most of the properties of these trapped quantum gases are governed by the interactions between particles in the condensate [1]. Over the past decade, there has been an investigation for realizing a new kind of quantum gases with the dipolar interaction, acting between particles having a permanent magnetic or electric dipole moment. The experimental realization of a BEC of $52 \mathrm{Cr}$ atoms $[2,3]$ at the University of Stuttgart in 2005 gave new impetus to the theoretical and numerical investigations on these novel dipolar quantum gases at low temperature. Recently more detailed and controlled experimental results have been obtained, illustrating the effects of phase separation in a multi-component BEC [4-6]. In these papers, the studies of the binary condensates were limited to the case of $s$-wave interactions, while recently the dipolar $\mathrm{BEC}$ has drawn a great deal of attention.

In this work, a numerical method for computing the ground state of the two-component dipolar BEC is considered, where one equation has dipole-dipole interactions and the other has only the usual $s$-wave contact interaction. However, since the dipole-dipole interactions are long range, anisotropic and partially attractive, and the computational cost in three dimensions is high, the nontrivial task of achieving and controlling the dipolar BEC is thus particularly challenging.

The two-component dipolar BEC, confined in a cylindrical trap, is described by two coupled Gross-Pitaevskii equations. As far as the dipolar interaction is concerned, a con- 
volution term is introduced [7-9] to modify the classical Gross-Pitaevskii equation, which results in the following differential-integral equations (1). Since the transition metal has a magnetic dipole interaction while the alkali metal has not, we take into account this factor in this system. We take $\mathrm{Cr}$ as component 1 and $\mathrm{Rb}$ as component 2 [10]. Then the GP equations for this system can be written as

$$
\left\{\begin{array}{l}
i \hbar \frac{\partial \varphi_{1}(\vec{r}, t)}{\partial t}=\left[-\frac{\hbar^{2}}{2 m} \nabla^{2}+V_{1}+U_{1}\left|\varphi_{1}\right|^{2}+U_{12}\left|\varphi_{2}\right|^{2}+V_{\mathrm{dip}}^{*}\left|\varphi_{1}\right|^{2}\right] \varphi_{1} \\
i \hbar \frac{\partial \varphi_{2}(\vec{r}, t)}{\partial t}=\left[-\frac{\hbar^{2}}{2 m} \nabla_{2}+V_{2}+U_{2}\left|\varphi_{2}\right|^{2}+U_{12}\left|\varphi_{1}\right|^{2}\right] \varphi_{2}
\end{array}\right.
$$

where $\varphi_{1}, \varphi_{2}$ are the wave functions of component 1 and 2, respectively. The inter-atomic and inter-component $s$-wave scattering interactions are described by $U_{j}(j=1,2)$ and $U_{12}$, respectively, with the following expressions [11]:

$$
U_{j}=\frac{4 \pi \hbar^{2} a_{j}}{m_{j}}, \quad U_{12}=\frac{2 \pi \hbar^{2} a_{j}}{m_{1} m_{2} /\left(m_{1}+m_{2}\right)} \quad(j=1,2),
$$

where $a_{j}$ is the scattering length of component $j$ and $a_{12}$ is that between component 1 and 2 . Here $\hbar$ is the Planck constant, $m_{j}$ is the mass of the atom of component $j$, and $V_{j}$ $(j=1,2)$ is the external trapping potential confining the gas. Generally, it is harmonic, that is, $V_{j}(\vec{r})=\frac{m_{j}}{2}\left(\omega_{j x}^{2} x^{2}+\omega_{j y}^{2} y^{2}+\omega_{j z}^{2} z^{2}\right)$ with $\omega_{j p}(p=x, y, z)$ represents the trap frequency in $x, y$, $z$ directions, respectively. The local mean-field $U_{j}\left|\varphi_{j}\right|^{2}$ represents the $s$-wave interaction. $V_{\text {dip }}$ is the long-range isotropic dipolar interaction potential between two dipoles, and it is defined by

$$
V_{\text {dip }}=\frac{\mu_{0} \mu_{\text {dip }}^{2}}{4 \pi} \cdot \frac{1-3(\vec{r} \cdot \vec{n})^{2} /|\vec{r}|^{2}}{|\vec{r}|^{3}}=\frac{\mu_{0} \mu_{\mathrm{dip}}^{2}}{4 \pi} \cdot \frac{1-3 \cos ^{2} \theta}{|\vec{r}|^{3}},
$$

where $\theta$ is the angle between the polarization axis $\vec{n}$ and the relative of two atoms (i.e., $\cos \theta=\vec{n} \cdot \vec{r} / r), r=|\vec{r}|=\sqrt{x^{2}+y^{2}+z^{2}}$. The wave function $\varphi_{i}(x, t)$ is normalized according to $\left\|\varphi_{i}\right\|^{2}=\int_{R^{3}}\left|\varphi_{i}(\vec{r}, t)\right|^{2} d \vec{r}=N_{i}(i=1,2)$, where $N_{i}$ is the number of the atoms in the dipolar BEC.

This paper is organized as follows. In Section 2, a numerical method for computing ground states is presented. In Section 3, numerical results are reported to verify the efficiency of this numerical method. Finally, some concluding remarks are drawn in Section 4.

\section{Numerical method for computing the ground states}

System (1) can be made dimensionless and simplified by adopting a unit system, where the units for length, time and energy are given by $a_{0}, 1 / \omega_{0}$, and $\hbar \omega_{0}$, respectively, with $a_{0}=\sqrt{\frac{\hbar}{m_{1} \omega_{0}}}, \omega_{0}=\min \left\{\omega_{j x}, \omega_{j y}, \omega_{j z}\right\}[12]$. By introducing the dimensionless variables $t^{\prime}=\frac{t}{\omega_{0}}$, $\overrightarrow{r^{\prime}}=\vec{r} / a_{0}, \varphi_{j}^{\prime}=a_{0}^{3 / 2} \varphi_{j}$, we obtain the dimensionless GP equations in 3D from (1) as follows:

$$
\left\{\begin{array}{l}
i \frac{\partial \varphi_{1}^{\prime}}{\partial t^{\prime}}=\left[-\frac{1}{2} \nabla^{2}+V_{1}^{\prime}+\beta_{11}\left|\varphi_{1}^{\prime}\right|^{2}+\beta_{12}\left|\varphi_{2}^{\prime}\right|^{2}+\lambda\left(V_{\mathrm{dip}}^{*}\left|\varphi_{1}^{\prime}\right|^{2}\right)\right] \varphi_{1}^{\prime}, \\
\left.i \frac{\partial \varphi_{2}^{\prime}}{\partial t^{\prime}}=\left[-\frac{1}{2} \nabla^{2}+a_{m} V_{2}^{\prime}+\beta_{21}\left|\varphi_{1}^{\prime}\right|^{2}+\beta_{22}\left|\varphi_{2}^{\prime}\right|^{2}\right)\right] \varphi_{2}^{\prime},
\end{array}\right.
$$

where $\beta_{11}=4 \pi a_{1} N_{1}, \beta_{12}=\frac{1+a_{m}}{a_{m}} 2 \pi a_{12} N_{2}, \beta_{21}=\frac{1+a_{m}}{a_{m}} 2 \pi a_{12} N_{1}, \beta_{22}=\frac{4 \pi a_{2}}{a_{m}} N_{2}, a_{m}=\frac{m_{2}}{m_{1}}, \lambda=$ $\frac{m_{1} N_{1} \mu_{0} \mu_{\text {dip }}^{2}}{3 \hbar^{2} a_{0}}, V_{\text {dip }}=\frac{3}{4 \pi} \cdot \frac{1-3 \cos ^{2} \theta}{r^{3}}$. In addition the wave functions in (2) satisfy $\int_{R^{3}}\left|\varphi_{1}^{\prime}\right|^{2}=1$, 
$\int_{R^{3}}\left|\varphi_{2}^{\prime}\right|^{2}=1$. By using the following formula [13]

$$
\frac{1}{r^{3}}\left(1-\frac{3(\vec{n} \cdot \vec{r})^{2}}{r^{2}}\right)=-\frac{3}{4 \pi} \delta(\vec{r})-\partial_{n n}\left(\frac{1}{r}\right)
$$

where $\delta(\vec{r})$ is the Dirac delta function and $\partial_{n n}=\vec{n} \cdot \nabla(\vec{n} \cdot \nabla)$, we can get

$$
V_{\text {dip }}^{*}\left|\varphi_{1}^{\prime}\right|^{2}=-\left|\varphi_{1}^{\prime}\right|^{2}-3 \partial_{n n}(\xi)
$$

where

$$
\xi(\vec{r}, t)=\frac{1}{4 \pi} \int_{R^{3}} \frac{1}{\left|\vec{r}-\overrightarrow{r^{\prime}}\right|} \cdot\left|\varphi_{1}^{\prime}\left(\overrightarrow{r^{\prime}}, t\right)\right|^{2} d \overrightarrow{r^{\prime}} .
$$

And it is easy to see

$$
\nabla^{2} \xi=-\left|\varphi_{1}^{\prime}(\vec{r}, t)\right|^{2}
$$

Plugging (4) into (2) and noticing (5) and (6), we can reformulate GPE (2) into the Schrödinger-Poisson type system

$$
\left\{\begin{array}{l}
i \frac{\partial \varphi_{1}}{\partial t}=\left[-\frac{1}{2} \nabla^{2}+V_{1}+\left(\beta_{11}-\lambda\right)\left|\varphi_{1}\right|^{2}+\beta_{12}\left|\varphi_{2}\right|^{2}-3 \lambda \partial_{n n}(\xi)\right] \varphi_{1}, \\
i \frac{\varphi_{2}}{\partial t}=\left[-\frac{1}{2} \nabla^{2}+V_{2}+\beta_{21}\left|\varphi_{1}\right|^{2}+\beta_{22}\left|\varphi_{2}\right|^{2}\right] \varphi_{2}, \\
\nabla^{2} \xi=-\left|\varphi_{1}(\vec{r}, t)\right|^{2},
\end{array}\right.
$$

by removing the symbol " in (2)-(6) to simplify the denotation. In practical computation, the whole space problem is usually truncated into a bounded computational domain $\Omega=$ $[a, b] \times[c, d] \times[e, f]$ with the homogeneous Dirichlet boundary condition. Let

$$
\begin{aligned}
& \Omega_{M K L}=\{(j, k, l) \mid j=1,2, \ldots, M-1, k=1,2, \ldots, K-1, l=1,2, \ldots, L-1\}, \\
& \Omega_{M K L}^{0}=\{(j, k, l) \mid j=0,1, \ldots, M, k=0,1, \ldots, K, l=0,1, \ldots, L\} .
\end{aligned}
$$

Choose the spatial mesh size as $h_{x}=\frac{b-a}{M}, h_{y}=\frac{d-c}{K}, h_{z}=\frac{f-e}{L}$, and define $x_{j}=a+j h_{x}, y_{k}=c+$ $k h_{y}, z_{l}=e+l h_{z},(j, k, l) \in \Omega_{M K L}^{0}$. Then denote the space $Y_{M K L}=\operatorname{span}\left\{\phi_{p q s}(\vec{r}),(p, q, s) \in \Omega_{M K L}\right\}$ with

$$
\phi_{p q s}(r)=\sin \left(u_{p}(x-a)\right) \sin \left(u_{q}(y-c)\right) \sin \left(u_{s}(z-e)\right)
$$

where $u_{p}=\frac{p \pi}{b-a}, u_{q}=\frac{q \pi}{d-c}, u_{s}=\frac{s \pi}{f-e}$.

To compute the ground state, an imaginary time method is adopted [14]. That is,

$$
\left\{\begin{array}{l}
\frac{\partial \varphi_{1}}{\partial t}=\left[\frac{1}{2} \nabla^{2}-V_{1}-\left(\beta_{11}-\lambda\right)\left|\varphi_{1}\right|^{2}-\beta_{12}\left|\varphi_{2}\right|^{2}+3 \lambda \partial_{n n}(\xi)\right] \varphi_{1} \\
\left.\frac{\partial \varphi_{2}}{\partial t}=\left[\frac{1}{2} \nabla^{2}-V_{2}-\beta_{21}\left|\varphi_{1}\right|^{2}-\beta_{22}\left|\varphi_{2}\right|^{2}\right)\right] \varphi_{2}, \\
\nabla^{2} \xi=-\left|\varphi_{1}(\vec{r}, t)\right|^{2}
\end{array}\right.
$$

We use the backward Euler method for time discretization and the sine-pseudospectral method for spatial derivatives. Then we obtain the following equations in a three- 


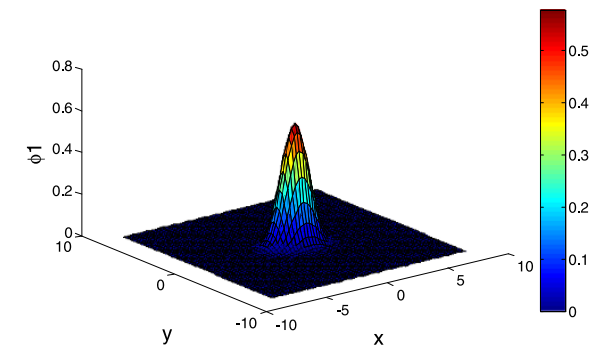

(a)

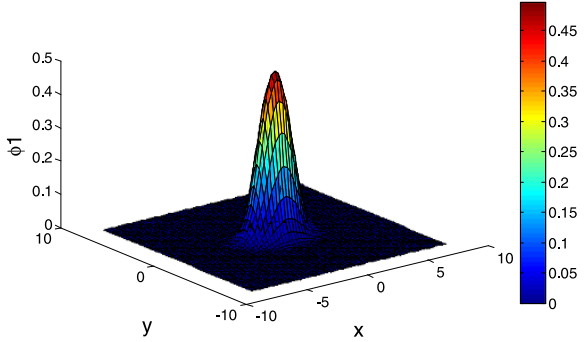

(c)

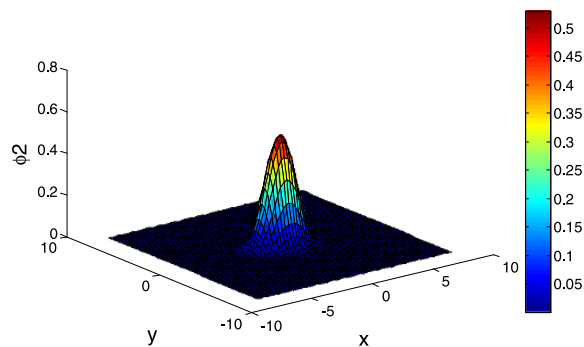

(b)

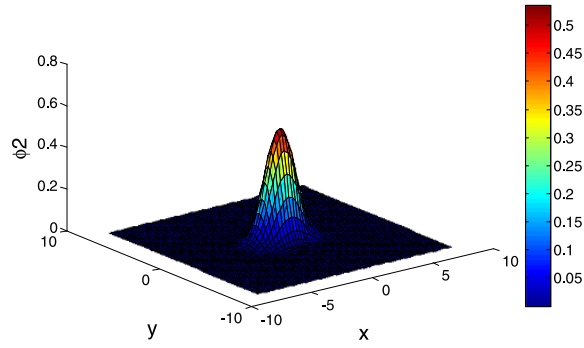

(d)

Figure 1 Ground state solutions for different parameter $\lambda$. (a) Surface for component $1, \varphi_{1}(x, y, z), z=0$ $\lambda=1 / 3$; (b) Surface for component $2, \varphi_{2}(x, y, z), z=0, \lambda=1 / 3$; (c) Surface for component $1, \varphi_{1}(x, y, z), z=0$, $\lambda=-1 / 3$; (d) Surface for component $2, \varphi_{2}(x, y, z), z=0, \lambda=-1 / 3$.

dimensional space. If we assume that

$$
\begin{aligned}
& \varphi_{1}\left(r, t_{n+1}\right)=\varphi_{1}^{n+1}(r)=\sum_{p=1}^{M-1} \sum_{q=1}^{K-1} \sum_{s=1}^{L-1} \alpha_{p q s} \phi_{p q s} \quad \text { and } \\
& \varphi_{2}\left(r, t_{n+1}\right)=\varphi_{2}^{n+1}(r)=\sum_{p=1}^{M-1} \sum_{q=1}^{K-1} \sum_{s=1}^{L-1} \alpha_{p q s}^{\prime} \phi_{p q s} . \\
& \left\{\begin{aligned}
& \frac{\varphi_{1 j k l}^{*}-\varphi_{1 j k l}^{n}}{\Delta t}= \frac{1}{2} \nabla_{h}^{2} \varphi_{1}^{*} \mid j k l-\left[V\left(x_{j}, y_{k}, z_{l}\right)+\left(\beta_{11}-\lambda\right)\left|\varphi_{1 j k l}^{n}\right|^{2}\right. \\
&\left.+\beta_{12}\left|\varphi_{2 j k l}^{n}\right|^{2}-3 \lambda \partial_{n n} \xi^{n} \mid j k l\right] \varphi_{1 j k l}^{*}, \\
& \frac{\varphi_{2 j k l}^{*}-\varphi_{2 j k l}^{n}}{\Delta t}= \frac{1}{2} \nabla_{h}^{2} \varphi_{2}^{*}||_{j k l}-\left[V\left(x_{j}, y_{k}, z_{l}\right)\right. \\
&\left.+\beta_{21}\left|\varphi_{2 j k l}^{n}\right|^{2}+\beta_{22}\left|\varphi_{1 j k l}^{n}\right|^{2}\right] \varphi_{2 j k l}^{*}, \quad t_{n} \leq t \leq t_{n+1}, \\
& \nabla^{2} \xi=-\left|\varphi_{1}(\vec{r}, t)\right|^{2},
\end{aligned}\right.
\end{aligned}
$$

with boundary conditions

$$
\begin{aligned}
& \varphi_{10 k l}^{*}=\varphi_{1 M k l}^{*}=\varphi_{1 j 0 l}^{*}=\varphi_{1 j K l}^{*}=\varphi_{1 j k 0}^{*}=\varphi_{1 j k L}^{*}=0, \quad(j, k, l) \in \Omega_{M K L}^{0}, \\
& \varphi_{20 k l}^{*}=\varphi_{2 M k l}^{*}=\varphi_{2 j 0 l}^{*}=\varphi_{2 j K l}^{*}=\varphi_{2 j k 0}^{*}=\varphi_{2 j k L}^{*}=0, \quad(j, k, l) \in \Omega_{M K L}^{0},
\end{aligned}
$$

and initial conditions

$$
\varphi_{1 j k l}^{0}=\varphi_{1,0}\left(x_{j}, y_{k}, z_{l}\right), \quad \varphi_{2 j k l}^{0}=\varphi_{2,0}\left(x_{j}, y_{k}, z_{l}\right), \quad(j, k, l) \in \Omega_{M K L}^{0},
$$




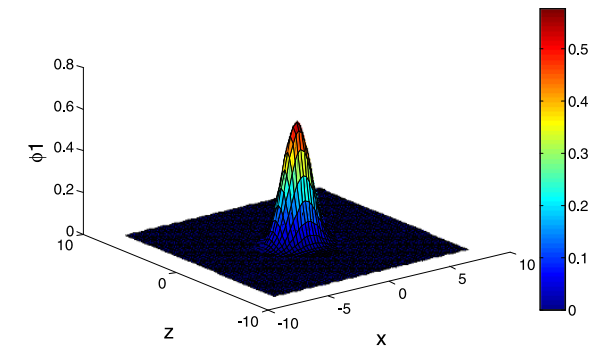

(a)

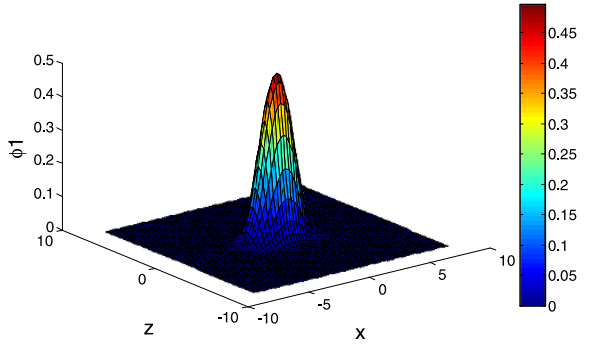

(c)

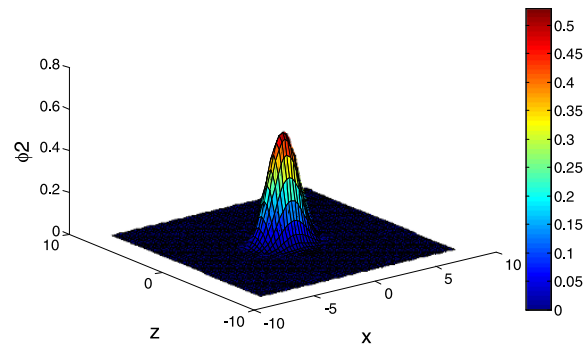

(b)

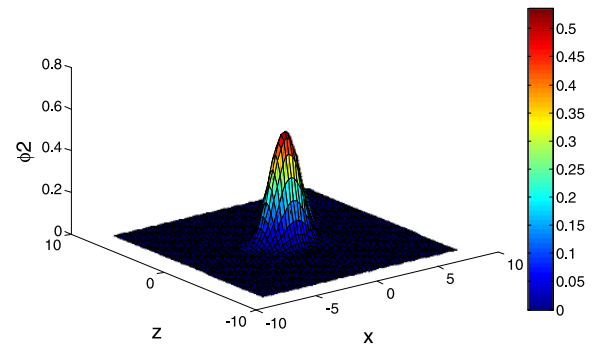

(d)

Figure 2 Ground state solutions for different parameter $\lambda$. (a) Surface for component $1, \varphi_{1}(x, y, z), y=0$, $\lambda=1 / 3$; (b) Surface for component $2, \varphi_{2}(x, y, z), y=0, \lambda=1 / 3$; (c) Surface for component $1, \varphi_{1}(x, y, z), y=0$, $\lambda=-1 / 3$; (d) Surface for component $2, \varphi_{2}(x, y, z), y=0, \lambda=-1 / 3$.

$$
\varphi_{1 j k l}^{n+1}=\frac{\varphi_{1 j k l}^{*}}{\left\|\varphi_{1}^{*}\right\|}, \quad \varphi_{2 j k l}^{n+1}=\frac{\varphi_{2 j k l}^{*}}{\left\|\varphi_{2}^{*}\right\|}, \quad(j, k, l) \in \Omega_{M K L},
$$

where $\left\|\varphi_{1}^{*}\right\|^{2}=h_{x} h_{y} h_{z} \sum_{j=1}^{M-1} \sum_{k=1}^{K-1} \sum_{l=1}^{L-1}\left|\varphi_{1 j k l}^{*}\right|^{2},\left\|\varphi_{2}^{*}\right\|^{2}=h_{x} h_{y} h_{z} \sum_{j=1}^{M-1} \sum_{k=1}^{K-1} \sum_{l=1}^{L-1}\left|\varphi_{2 j k l}^{*}\right|^{2}$; $\nabla_{h}^{2} \varphi^{n}$ and $\partial_{n n} \xi^{n}$ are sine pseudo-spectral approximations of $\nabla^{2} \varphi$ and $\partial_{n n} \xi$ at time $t=t_{n}$, respectively, defined for $(p, q, s) \in \Omega_{M K L}$ as

$$
\begin{aligned}
& \left.\nabla^{2} \varphi_{1}^{n+1}(r)\right|_{j k l}=-\sum_{p q s}\left(u_{p}^{2}+u_{q}^{2}+u_{s}^{2}\right) \alpha_{p q s} \sin \left(\frac{j p \pi}{M}\right) \sin \left(\frac{k q \pi}{K}\right) \sin \left(\frac{l s \pi}{L}\right), \\
& \left.\nabla^{2} \varphi_{2}^{n+1}(r)\right|_{j k l}=-\sum_{p q s}\left(u_{p}^{2}+u_{q}^{2}+u_{s}^{2}\right) \alpha_{p q s}^{\prime} \sin \left(\frac{j p \pi}{M}\right) \sin \left(\frac{k q \pi}{K}\right) \sin \left(\frac{l s \pi}{L}\right), \\
& \left.\partial_{n n} \xi^{n}(r)\right|_{j k l}=-\sum_{p q s}\left(u_{p}^{2}+u_{q}^{2}+u_{s}^{2}\right) \beta_{p q s} \sin \left(\frac{j p \pi}{M}\right) \sin \left(\frac{k q \pi}{K}\right) \sin \left(\frac{l s \pi}{L}\right)=-\left|\varphi_{1 p q s}(r)\right|^{2} .
\end{aligned}
$$

The discrete sine transform coefficients of the vector $\left.\phi_{p q s}(r)\right|_{\left(x_{j} ; y_{k}, z_{l}\right)}$ for $(p, q, s) \in \Omega_{M K L}$ are

$$
\begin{aligned}
& \alpha_{p q s}=\frac{8}{M K L} \sum_{j=1}^{M-1} \sum_{k=1}^{K-1} \sum_{l=1}^{L-1} \varphi_{1 j k l}^{n+1} \sin \left(\frac{j p \pi}{M}\right) \sin \left(\frac{k q \pi}{K}\right) \sin \left(\frac{l s \pi}{L}\right), \\
& \alpha_{p q s}^{\prime}=\frac{8}{M K L} \sum_{j=1}^{M-1} \sum_{k=1}^{K-1} \sum_{l=1}^{L-1} \varphi_{2 j k l}^{n+1} \sin \left(\frac{j p \pi}{M}\right) \sin \left(\frac{k q \pi}{K}\right) \sin \left(\frac{l s \pi}{L}\right),
\end{aligned}
$$




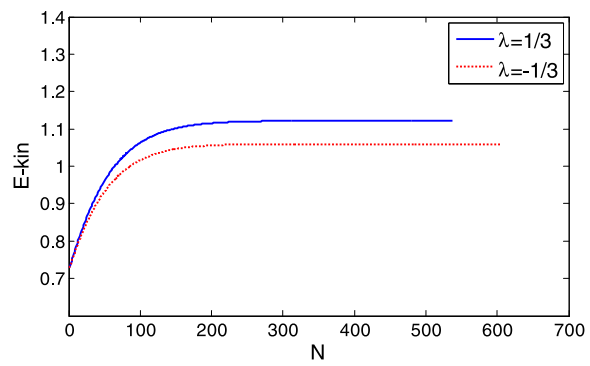

(a)

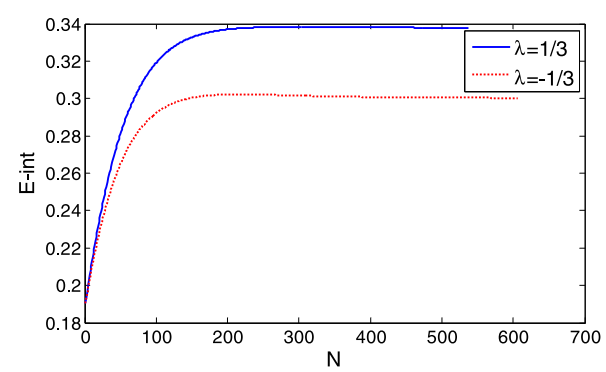

(c)

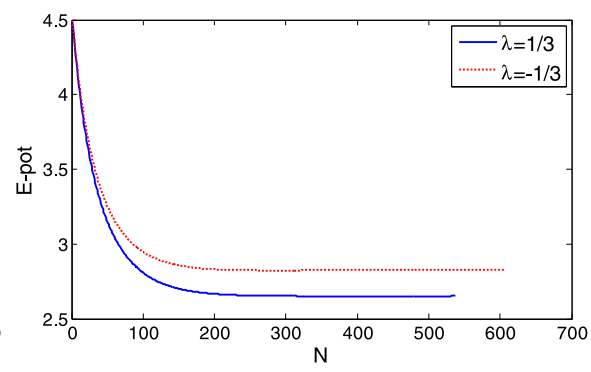

(b)

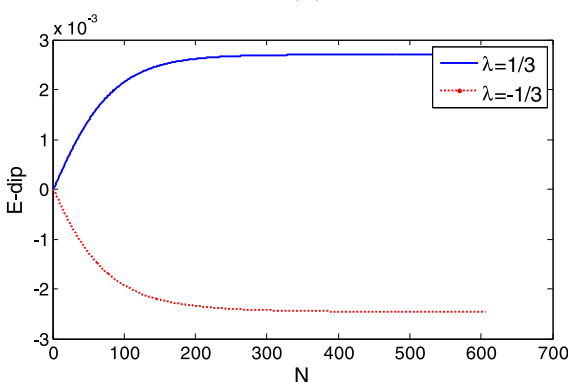

(d)

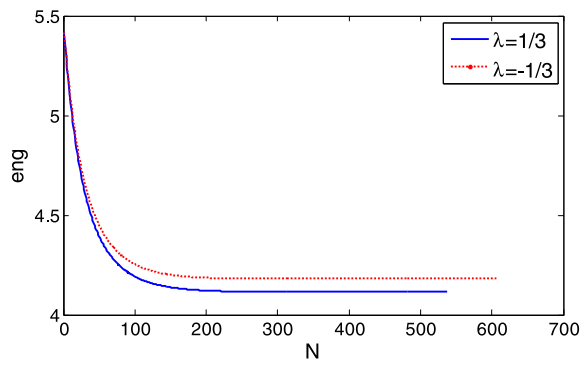

(e)

Figure 3 The energy evolution according to the time step $\boldsymbol{N}$. (a) Kinetic energy; (b) Potential energy; (c) Interaction energy; (d) Dipole energy; (e) The total energy.

$$
\beta_{p q s}=\frac{1}{u_{p}^{2}+u_{q}^{2}+u_{s}^{2}} \frac{8}{M K L} \sum_{j=1}^{M-1} \sum_{k=1}^{K-1} \sum_{l=1}^{L-1}\left|\varphi_{1 j k l}^{n+1}\right|^{2} \sin \left(\frac{j p \pi}{M}\right) \sin \left(\frac{k q \pi}{K}\right) \sin \left(\frac{l s \pi}{L}\right) .
$$

Linear system (7) can be iteratively solved in a phase space very efficiently via discrete sine transform under the conditions $\max _{j, k, l}\left|\varphi_{1 j k l}^{n+1}-\varphi_{1 j k l}^{n}\right|<10^{-6}$ and $\max _{j, k, l}\left|\varphi_{2 j k l}^{n+1}-\varphi_{2 j k l}^{n}\right|<10^{-6}$.

\section{Numerical results}

Example 1 Consider the ground state of the BEC with $V(r)=\frac{1}{2}\left(x^{2}+2 y^{2}+2 z^{2}\right)$. The initial condition is given as follows:

$$
\left\{\begin{array}{l}
\varphi_{1,0}=\varphi_{1}(x, y, z, 0)=\pi^{-3 / 4} \gamma_{x}^{1 / 2} \gamma_{z}^{1 / 4} e^{-\frac{1}{2}\left(\gamma_{x}\left(x^{2}+y^{2}\right)+\gamma_{z} z^{2}\right)}, \\
\varphi_{2,0}=\varphi_{2}(x, y, z, 0)=\pi^{-3 / 4} \gamma_{x}^{1 / 2} \gamma_{z}^{1 / 4} e^{-\frac{1}{2}\left(\gamma_{x}\left(x^{2}+y^{2}\right)+\gamma_{z} z^{2}\right)},
\end{array} \quad(x, y, z) \in R^{3} .\right.
$$

Here, $\gamma_{x}=\frac{\omega_{x}}{2 \omega_{0}}, \gamma_{z}=\frac{\omega_{z}}{2 \omega_{0}}, \omega_{0}=\min \left\{\omega_{j x}, \omega_{j y}, \omega_{j z}\right\}$. We solve this system on $[-8,8]^{3}$ with $h_{x}=$ $h_{y}=h_{z}=1 / 4$ and $\Delta t=0.005$. 


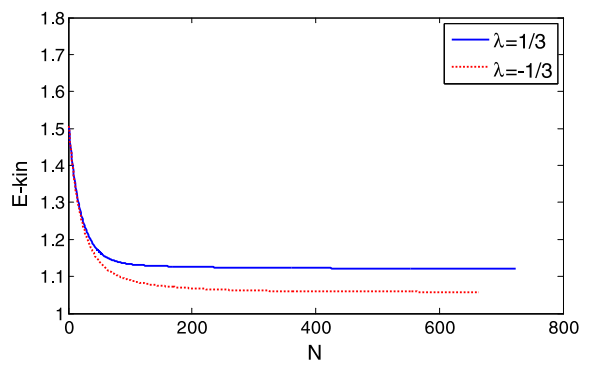

(a)

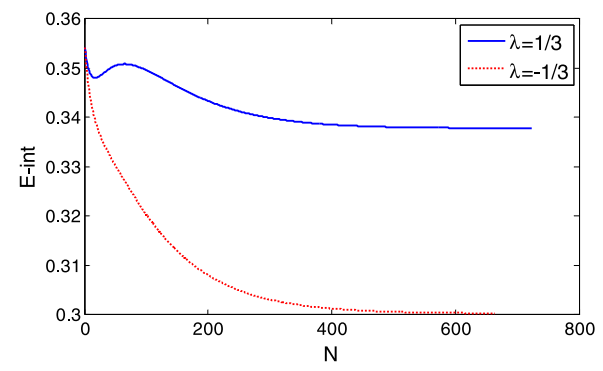

(c)

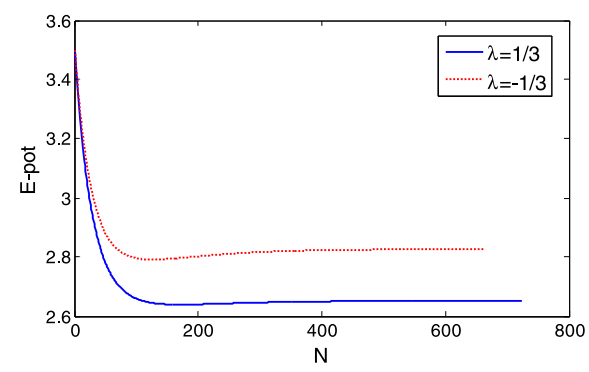

(b)

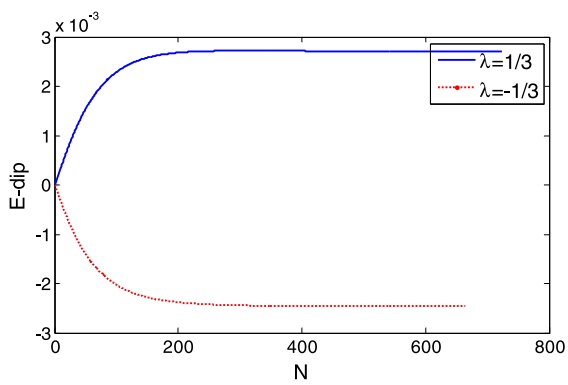

(d)

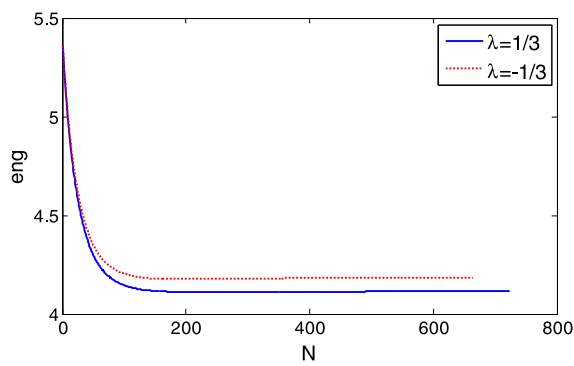

(e)

Figure 4 The energy evolution according to the time step $\boldsymbol{N}$. (a) Kinetic energy; (b) Potential energy; (c) Interaction energy; (d) Dipole energy; (e) The total energy.

Figure 1 shows the results for the case of $\gamma_{x}=\gamma_{z}=1$.

Example 2 Solve the ground state problem for a dipolar BEC with $V(r)=\frac{1}{2}\left(x^{2}+2 y^{2}+2 z^{2}\right)$.

The initial condition is

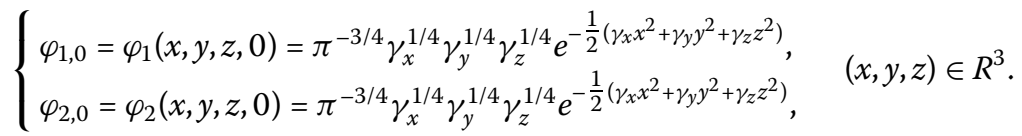

Figure 2 shows the results for the case of $\gamma_{x}=2, \gamma_{y}=8, \gamma_{z}=1$.

\section{Conclusion}

An efficient numerical method is presented for computing the ground states of dipolar Bose-Einstein condensates based on two coupled three-dimensional Gross-Pitaevskii equations, where one equation has a dipole-dipole interaction potential and the other one has only the usual $s$-wave contact interaction. Using equality (3), we can reformulate the 
GPE for dipolar BEC into a Gross-Pitaevskii-Poisson type system. Numerical examples are given to show the efficiency of our method. In all cases, total energy decreases (Figure 3 and Figure 4). The results agree with the previous work [15]. Numerical results are given to demonstrate the efficiency of our numerical method. And dynamics will be the latter part of our work to be carried out.

Competing interests

The authors declare that they have no competing interests.

\section{Authors' contributions}

SQL established the scheme, performed the numerical examples in Section 3 and drafted the manuscript. XGL designed the study. DYH established the model and helped to inspect the manuscript. All authors read and approved the final manuscript.

\section{Acknowledgements}

This work was supported by Natural Science Foundation of China (No. 11171032) and Beijing Municipal Education Commission (Nos. KM201110772017, 71D09111003).

Received: 15 March 2013 Accepted: 25 June 2013 Published: 8 July 2013

\section{References}

1. Pitaevskii, L, Stringari, S: Bose-Einstein Condensation. Oxford University, New York (2003)

2. Griesmaier, A, Werner, J, Hensler, S, Stuhler, J, Pfau, T: Bose-Einstein condensation of chromium. Phys. Rev. Lett. 94, Article ID 160401 (2005)

3. Stuhler, J, Griesmaier, A, Koch, T, Fattori, M, Pfau, T, Giovanazzi, S, Pedri, P, Santos, L: Observation of dipole-dipole interaction in a degenerate quantum gas. Phys. Rev. Lett. 95, Article ID 150406 (2005)

4. Schweikhard, V, Coddington, I, Engels, P, Tung, S, Cornell, EA: Phys. Rev. Lett. 93, 210403 (2004)

5. Mertes, KM, Merrill, JW, Carretero-González, R, Frantzeskakis, DJ, Kevrekidis, PG, Hall, DS: Phys. Rev. Lett. 99, 190402 (2007)

6. Papp, SB, Pino, JM, Wieman, CE: Phys. Rev. Lett. 101, 040402 (2008)

7. Yi, S, You, L: Trapped atomic condensates with anisotropic interactions. Phys. Rev. A 61, 041604(R) (2000)

8. Yi, S, You, L: Trapped condensates of atoms with dipole interactions. Phys. Rev. A 63, Article ID 053607 (2001)

9. Yi, S, You, L: Calibrating dipolar interaction in an atomic condensate. Phys. Rev. Lett. 92, Article ID 193201 (2004)

10. Xi, K-T, Li, J, Shi, D-N: Phase separation of a two-component dipolar Bose-Einstein condensate in the quasi-one-dimensional and quasi-two-dimensional regime. Phys. Rev. A 84, Article ID 013619 (2011)

11. Pethick, CJ, Smith, H: Bose-Einstein Condensation in Dilute Gases. Cambridge University Press, Cambridge, (2008)

12. Hua, D-Y, Li, X-G, Zhu, J: A mass conserved splitting method for the nonlinear Schrödinger equation. Adv. Differ. Equ. 2012, 85 (2012)

13. Bao, W, Cai, Y: Mathematical theory and numerical methods for Bose-Einstein condensation. Kinet. Relat. Models 6(1), 1-135 (2013)

14. Chiofalo, ML, Succi, S, Tosi, MP: Ground state of trapped interacting Bose-Einstein condensates by an explicit imaginary time algorithm. Phys. Rev. E 62, 7438-7444 (2000)

15. Parker, NG, Ticknor, C, Martin, AM, O'Dell, DHJ: Structure formation during the collapse of a dipolar atomic Bose-Einstein condensate. Phys. Rev. A 79, Article ID 013617 (2009)

doi:10.1186/1687-1847-2013-204

Cite this article as: Li et al.: The numerical method for computing the ground state of the two-component dipolar Bose-Einstein condensate. Advances in Difference Equations 2013 2013:204.

\section{Submit your manuscript to a SpringerOpen ${ }^{\circ}$ journal and benefit from:}

- Convenient online submission

Rigorous peer review

- Immediate publication on acceptance

- Open access: articles freely available online

- High visibility within the field

- Retaining the copyright to your article 